\title{
How often is a work-up for Legionella pursued in patients with pneumonia? A retrospective study
}

\author{
Brian Hollenbeck', Irene Dupont ${ }^{2}$ and Leonard A Mermel ${ }^{2,3^{*}}$
}

\begin{abstract}
Background: It is unclear how often patients with pneumonia are assessed for Legionella in endemic areas. Additionally, the sensitivity of the IDSA/ATS criteria for recommended Legionella testing is undefined.
\end{abstract}

Methods: We performed a single-center, retrospective study of patients diagnosed with Legionella pneumonia at our hospital to determine: 1) how often Legionella diagnostic testing is obtained on patients with pneumonia at the time of hospitalization or when pneumonia developed during hospitalization; and 2) how often patient's with Legionella pneumonia met at least one of the five criteria in the IDSA/ATS guidelines recommending a work-up for Legionella. Patients with Legionella pneumonia were identified using an infection control software program. Medical records of these patients were then reviewed.

Results: Thirty-five percent of patients with a discharge diagnosis of pneumonia had Legionella urine antigen testing and/or a Legionella culture performed. Forty-four percent of patients who had a bronchoscopic specimen sent for microbiologic testing had a Legionella culture performed on the bronchoscopic specimen and/or Legionella urine antigen testing. Of 37 adult patients with Legionella pneumonia, 22 (59\%) met the IDSA-ATS criteria recommending Legionella testing.

Conclusion: Following current recommendations for Legionella testing missed $41 \%$ of Legionella cases in adults in our single-center study. A work-up for Legionella (i.e., urine antigen test and/or culture) was performed in less than half of patients who have a bronchoscopic specimen sent for microbiologic testing.

Keywords: Legionella, Legionnaires' disease, pneumonia

\section{Background}

In the Infectious Diseases Society of America (IDSA) and American Thoracic Society (ATS) communityacquired pneumonia guidelines [1], Legionella pneumophila urine antigen testing is recommended for patients with any of the following: severe pneumonia requiring intensive care unit (ICU) admission, failure of outpatient antibiotics, active alcohol abuse, history of travel within the previous two weeks, or pleural effusion. For every patient with community-acquired pneumonia, empiric treatment of Legionella is recommended using a macrolide or respiratory fluoroquinolone [1]. However, making a diagnosis of Legionella allows the practitioner to more accurately define the choice and duration of

\footnotetext{
* Correspondence: Imermel@lifespan.org

2Department of Epidemiology and Infection Control, Rhode Island Hospital, 593 Eddy Street, Providence RI 02903 USA

Full list of author information is available at the end of the article
}

antimicrobial therapy and such testing assists public health officials in detecting Legionella outbreaks in community and healthcare settings.

The aims of this study were to determine: 1 ) how often Legionella diagnostic testing is obtained by physicians managing all patients with pneumonia; 2) how often Legionella testing is obtained for patients who have an heightened acuity of illness prompting bronchoscopic evaluation as a diagnostic test for possible pneumonia; and 3) the sensitivity of current IDSA/ATS guidelines for recommending Legionella testing in patients with pneumonia.

\section{Methods}

The TII ECLYPSIS software program (Chicago, IL), which identifies specific ICD-9 codes and billing data, was used to retrospectively determine the number of patients with a primary or secondary diagnosis of

\section{Biomed Central}


pneumonia and to determine the number of patients who had a bronchoscopy and a bronchoscopic specimen sent for microbiologic testing between October 1, 2007 and March 31, 2009. The same software program was used to identify the number of patients who had urine Legionella antigen testing (BinaxNow, Inverness Medical Professional Diagnostics, Princeton, NJ) or who had a Legionella culture using standard microbiological methods [2].

The TheraDoc ${ }^{\circledR}$ software program (Hospira, Lake Forest, IL) was used by the Department of Epidemiology and Infection Control at Rhode Island Hospital to prospectively identify patients at least 18 years of age with community or hospital-acquired Legionella pneumonia between January 1, 2005 and December 31, 2009 in our 719 bed tertiary care, university-affiliated hospital in Southern New England. For the purposes of this study, we retrospectively assessed this data and performed chart reviews as noted below. Each patient was included only once; incarcerated patients were excluded due to IRB restrictions.

Chart reviews were retrospectively performed by one of the authors $(\mathrm{BH})$ to determine demographic, laboratory, and clinical features of each Legionella case, including the five criteria for Legionella testing noted in the IDSA/ATS guideline as follows: history of alcohol abuse, recent travel within 2 weeks, pleural effusion upon admission, admission to an intensive care unit for pneumonia, and failure of outpatient antibiotics. Both electronic and paper charts were reviewed in a systematic fashion. Hyponatremia was defined as sodium $<130$ $\mathrm{mEq} / \mathrm{L}$. This study was approved by the Rhode Island Hospital IRB committee (IRB Registration \# 00000396). All authors had full access to all the data in the study and take responsibility for the integrity of the data and accuracy of the data analysis.

\section{Results}

There were 3,982 patients of any age with a primary or secondary diagnosis of pneumonia between October 1, 2007 and March 31, 2009. Of these, 1,406 patients (35\%) had a Legionella urine antigen test and/or Legionella culture as follows: 1251 had a urine antigen test alone; 49 had a Legionella culture alone; and 106 had both a Legionella culture and urine antigen test. During the same time period, 626 patients underwent bronchoscopy and had bronchoscopic specimens sent to the microbiology laboratory for culture, 277 (44\%) of whom had Legionella testing done as follows: 122 had a Legionella culture alone; 31 had a urine antigen test alone; and 124 had both a Legionella culture and urine antigen test.

Forty-one adult patients with Legionella pneumonia were identified between January 1, 2005 and December $31,2009,37$ of which were included in the study (Table
1). Reasons for exclusion were as follows: under 18 years of age ( 2 cases); incarcerated patient (1 case); and Legionella pneumonia was diagnosed and treated at an outside hospital but the Legionella urine antigen test remained positive when retested at admission to our hospital ( 1 case). There were 6, 5, 5, 12, and 9 Legionella cases in 2005 through 2009, respectively. All 37 cases were $L$. pneumophila serogroup 1 . Thirty-six cases were diagnosed by urine antigen testing alone; one case had a positive urine antigen test and a positive Legionella culture from a bronchoscopic specimen. Thirty-three of the 37 cases $(89 \%)$ met CDC surveillance criteria for community-acquired Legionnaires' disease; the remaining four cases $(11 \%)$ met criteria for possible hospitalacquired pneumonia. All 4 patients with possible hospital-acquired Legionella pneumonia were immunosuppressed. Twenty-two of 37 cases (59\%) met at least one of the five IDSA/ATS criteria recommending Legionella testing as follows: 14 (38\%) were admitted to an ICU; 7 (19\%) had a pleural effusion on initial chest radiograph; $6(16 \%)$ had a history of alcohol abuse; 4 (11\%) failed outpatient antibiotics prior to admission; and $1(3 \%)$ had a history of recent travel. Among the 37 cases, the most common findings were: immunocompromised status, 24 cases (65\%); hyponatremia, 24 cases (65\%); diarrhea 14 cases (38\%), and ICU admission, 14 cases (38\%). Chart review revealed that liver function testing was not performed in 8 patients and presence or absence of diarrhea was not recorded in 2 patients.

\section{Discussion}

Thirty-five percent of patients with a discharge diagnosis of pneumonia between October 1, 2007 and March 31, 2009 were tested for Legionella. Of patients whose acuity of illness led to the need for bronchoscopy and who had a bronchoscopic specimen sent for microbiologic testing, $44 \%$ had Legionella testing by urine antigen or culture [3]. These results suggest that available methodologies to diagnose Legionella may be underutilized in regions of the USA where Legionella is endemic.

Of 37 cases of Legionnaires' disease diagnosed in our hospital over 5 years, 22 (59\%) met IDSA/ATS criteria for Legionella testing. Thus, $41 \%$ of Legionella cases may have gone undiagnosed if testing was only done according to the IDSA/ATS recommendations. Of the 5 clinical features recommended by IDSA/ATS guidelines, admission to ICU and the presence of a pleural effusion were most helpful in identifying patients with Legionella pneumonia. The low number of patients presenting with antibiotic failure may reflect the widespread use of macrolides and quinolones by primary care providers.

Nearly all of our cases were community-acquired and all cases were L. pneumophila serogroup 1 . Thirty-six of our 37 cases were diagnosed solely by urine antigen testing. 
Table 1 Characteristics of Adult Patients with Legionella Pneumonia, 2005-2009

\begin{tabular}{|c|c|c|c|}
\hline & $\begin{array}{l}\text { Patients with Legionella that met IDSA/ } \\
\text { ATS criteria for Legionella testing }\end{array}$ & $\begin{array}{l}\text { Patients with Legionella that did not meet } \\
\text { IDSA/ATS criteria for Legionella testing }\end{array}$ & $\begin{array}{c}\text { All patients with } \\
\text { Legionella } \\
\text { pneumonia }\end{array}$ \\
\hline Male & 15 & 9 & $24(65 \%)$ \\
\hline Recent travel & 1 & 0 & $1(3 \%)$ \\
\hline History of alcohol abuse & 6 & 0 & $6(16 \%)$ \\
\hline Anti-TNF therapy & 1 & 1 & $2(5 \%)$ \\
\hline Daily steroid use & 0 & 1 & $1(3 \%)$ \\
\hline Cancer & 4 & 4 & $8(22 \%)$ \\
\hline Diabetes & 5 & 6 & $11(29 \%)$ \\
\hline COPD & 5 & 4 & $9(24 \%)$ \\
\hline $\begin{array}{l}\text { Solid-organ transplant } \\
\text { recipient }\end{array}$ & 0 & 1 & $1(3 \%)$ \\
\hline HIV infection & 1 & 0 & $1(3 \%)$ \\
\hline $\begin{array}{l}\text { Receipt of antibiotics } \\
\text { prior to hospitalization }\end{array}$ & 4 & 0 & $4(11 \%)$ \\
\hline $\begin{array}{l}\text { Pleural effusion present } \\
\text { at hospital admission }\end{array}$ & 7 & 0 & $7(19 \%)$ \\
\hline ICU admission & 14 & 0 & $14(38 \%)$ \\
\hline $\begin{array}{l}\text { Abnormal liver function } \\
\text { tests }\end{array}$ & 15 & 5 & $20(54 \%)$ \\
\hline $\begin{array}{l}\text { Hyponatremia (Sodium < } \\
130 \mathrm{mEq} / \mathrm{L} \text { ) }\end{array}$ & 9 & 4 & $13(35 \%)$ \\
\hline Crude mortality & 6 & 0 & $6(16 \%)$ \\
\hline
\end{tabular}

$\mathrm{SD}$, standard deviation; TNF, tumor necrosis factor; COPD, chronic obstructive pulmonary disease; abnormal liver function test defined as above reference range; Crude mortality defined as in-hospital death or discharge to hospice care with impending death.

This suggests that cases of Legionnaires' disease due to other serogroups may have been missed because Legionella culture was underutilized. The CDC estimates 2.5 to 5.8 Legionella cases/100,000 population yearly in the USA $[4,5]$. In Rhode Island, epidemiologic data on Legionnaires' disease are available from 2004 to 2008 [6], during which time the annual incidence ranged from 2.0 to 4.7 cases/ 100,000 population. There was a trend towards an increasing incidence of Legionella pneumonia in Rhode Island during this time period similar to national data suggesting increasing incidence of Legionnaires' disease from 1990 to 2005 , with the most pronounced rise in noted in the Northeastern USA [7].

Limitations of our study reflect a single-center experience. As such, the incidence of Legionnaires' disease in our geographic location may not be representative of other settings. Since we used coded data to determine the number of patients with a discharge diagnosis of pneumonia, we may have underestimated or overestimated the true number of pneumonia cases at our hospital. We combined community and possible hospitalacquired cases to assess the sensitivity of the IDSA/ATS guidelines although the guidelines specifically address community-acquired pneumonia. However, the sensitivity of the recommended testing for Legionella in our 33 community-acquired cases was $58 \%$. Lastly, education programs and hospital-specific diagnostic algorithms in other institutions may promote more widespread Legionella testing.

A number of studies have searched for clinical features that are specific for Legionnaires' disease without particular success $[8,9]$. In endemic areas such as the Eastern USA, where pretest probability is higher [7], our study suggests the possible need to extend urine Legionella antigen testing beyond what currently occurs, as suggested by the number of Legionella pneumonia cases that did not meet the IDSA/ATS criteria for testing, the proportion of pneumonia cases that are currently being tested for Legionnaires' disease, and the possibility of rising incidence. The best strategy at the present time may be to screen all cases of community-acquired pneumonia requiring hospital admission in endemic locales but the cost-effectiveness of such a strategy first needs to be assessed. Such a testing program will likely identify more Legionella pneumonia cases, thereby focusing antimicrobial therapy. Additionally, such testing would better assist public health departments by improving outbreak detection, and it may lead to a better understanding of the epidemiology of Legionnaires' disease and its prevention.

\section{Conclusions}

A diagnostic work-up for Legionella (i.e., urine antigen testing or culture of respiratory specimens) is performed 
in less than half of patients with pneumonia in our endemic region. If Legionella testing was curtailed to those recommended in the IDSA/ATS communityacquired pneumonia guidelines, the diagnosis of Legionella pneumonia would have been missed in $41 \%$ of our cases. Thus, Legionella pneumonia may be underdiagnosed and more widespread Legionella testing should be considered to better delineate the choice and duration of antimicrobial therapy and to assist in uncovering clusters of Legionella in the community or healthcare setting.

\section{Acknowledgements and funding}

We acknowledge the following individuals for their assistance in data collection: J. Jefferson, RN, MPH, other members of the Department of Epidemiology and Infection Control at Rhode Island Hospital, Kerry Puniello and Sue Simundson MHA. This study was unfunded.

\section{Author details}

'Department of Medicine, Rhode Island Hospital and The Warren Alpert Medical School of Brown University, 593 Eddy Street, Providence, RI 02903 USA. ${ }^{2}$ Department of Epidemiology and Infection Control, Rhode Island Hospital, 593 Eddy Street, Providence RI 02903 USA. ${ }^{3}$ Division of Infectious Diseases, Rhode Island Hospital, 593 Eddy Street, Providence, RI 02903 USA.

\section{Authors' contributions}

The study idea was developed by LM and BH. Data entry and analysis were performed by $\mathrm{ID}$ and $\mathrm{BH}$. $\mathrm{BH}$ wrote the first draft of the manuscript which was extensively reviewed by LM and ID. All authors have read and approved the final manuscript.

\section{Competing interests}

The authors declare that they have no competing interests.

Received: 21 January 2011 Accepted: 7 September 2011

Published: 7 September 2011

\section{References}

1. Mandell LA, Wunderink RG, Anzueto A, Bartlett JG, Campbell GD, Dean NC, Dowell SF, File TM Jr, Musher DM, Niederman MS, Torres A, Whitney CG Infectious Diseases Society of America; American Thoracic Society. Infectious Diseases Society of America/American Thoracic Society Consensus Guidelines on the Management of Community-Acquired Pneumonia in Adults. Clin Infect Dis 2007, 44(Suppl 2):s27-72.

2. Pasculle W, McDevitt D: Legionella cultures. In Clinical Microbiology Procedures Handbook.. 2 edition. Edited by: Garcia LS. Washington, DC: ASM Press; 2007:3.11, 4.1-15.

3. Yzerman EP, den Boer JW, Lettinga KD, Schellekens J, Dankert J, Peeters M: Sensitivity of three urinary antigen tests associated with clinical severity in a large outbreak of legionnaires' disease in the Netherlands. J Clin Microbiol 2002, 40:3232-3236.

4. Centers for Disease Control and Prevention (CDC): Legionellosis Resource Site (Legionnaires' Disease and Pontiac Fever).[http://www.cdc.gov/ legionella/index.htm], Accessed 13 October 2010.

5. U.S. Census Bureau: U.S. POPClock Projection.[http://www.census.gov/ population/www/popclockus.html], Accessed 13 October 2010.

6. State of Rhode Island Department of Health: Office of Communicable Diseases.[http://www.health.ri.gov/disease/communicable/legionellosis.php], Accessed 13 October 2010.

7. Neil K, Berkelman R: Increasing incidence of legionellosis in the United States 1990-2005: changing epidemiologic trends. Clin Infect Dis 2008, 47:591-599.

8. Granados A, Podzamczer D, Gudiol F, Manresa F: Pneumonia due to Legionella pneumophila and pneumococcal pneumonia: similarities and differences on presentation. Eur Respir J 1989, 2:130-134.
9. Roig J, Aguilar X, Ruiz J, Domingo C, Mesalles E, Manterola J, Morena J: Comparative study of Legionella pneumophila and other nosocomialacquired pneumonias. Chest 1991, 99:344-350.

\section{Pre-publication history}

The pre-publication history for this paper can be accessed here: http://www.biomedcentral.com/1471-2334/11/237/prepub

\section{doi:10.1186/1471-2334-11-237}

Cite this article as: Hollenbeck et al.: How often is a work-up for Legionella pursued in patients with pneumonia? A retrospective study. BMC Infectious Diseases 2011 11:237.

\section{Submit your next manuscript to BioMed Central and take full advantage of:}

- Convenient online submission

- Thorough peer review

- No space constraints or color figure charges

- Immediate publication on acceptance

- Inclusion in PubMed, CAS, Scopus and Google Scholar

- Research which is freely available for redistribution 